\title{
Non Syndromic Generalised Taurodontism in Primary and Permanent teeth -A Case Report
}

\author{
Bharath $^{1}$. KP, Priya ${ }^{2}$, Poornima .P. ${ }^{3}$, Neena I.E ${ }^{4}$
}

${ }^{1-4}$ Reader, ${ }^{2}$ PG Student, ${ }^{3}$ Professor

Dept. of Pedodontics, College of Dental Sciences, Davangere

\section{Abstract:}

Taurodontism is a rare dental anomaly defined as a change in shape of the tooth caused by the failure of Hertwig's epithelial sheath to invaginate at the proper horizontal level. The characteristic features of this anomaly are an enlarged pulp chamber with apical displacement of the pulpal floor, and no constriction at the level of the Cemento-enamel junction. They occur most frequently as an isolated anomaly or it may be associated with certain syndromes. Permanent dentition is more commonly affected than deciduous dentition, seen unilaterally or bilaterally, and in any combination of teeth or quadrants. This case report describes a case of generalised taurodontism involving all the deciduous molars and permanent molars in a 7 years old male patient.

Keywords: Bilateral, Deciduous teeth, Pulp chamber Taurodontism

\section{Introduction}

Taurodontism is a morpho-anatomical change in the shape of the tooth characterized by enlargement of the pulp chamber at the expense of the roots and apical displacement of furcation areas. The bifurcation / trifurcation may only be few millimetres above the apices of the roots ${ }^{1}$ (Figure 1). Taurodontism was first described by Gorjanovic - Kramberger in 1908 but it was later Sir Arthur Keith who stated the term 'taurodontism' in 1913. The term 'taurodontism' was coined from the Latin term 'tauros', which means 'bull' and the Greek term 'odus', which means 'tooth' ${ }^{2}$. According to Witkop taurodontism is defined as those teeth having large pulp chambers with apically displaced bifurcation or trifuracation, resulting in pulp chamber with greater apicoocclusal height than the normal teeth and lack of cervical constriction. The prevalence varies from $2.5-3.2 \%$ in permanent teeth while in deciduous teeth it is $0.54 \%{ }^{3}$.

The conditions leading to taurodontism are a mutation, a primitive pattern, a retrograde character, an atavistic feature, an X-linked trait, familial or an autosomal dominant trait ${ }^{3}$. Taurodontism occurs as an isolated anomaly, but it is said to be associated with several developmental syndromes and anomalies including Down's syndrome, Ectodermal dysplasia, Klinefelter

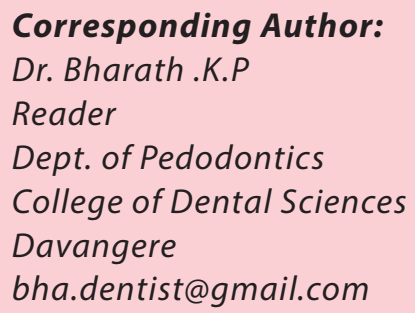

syndrome, Tricho-dento-osseous syndrome, Mohr syndrome, Wolf-Hirschhorn syndrome,Williams syndorme and Lowe syndrome ${ }^{4}$. Various Theories regarding the pathogenesis of taurodontic root formation are put forward: an unusual developmental pattern, a delay in the calcification of pulpal chamber, an odontoblastic deficiency, an alteration in Hertwig's epithelial root sheath ${ }^{1}$.

According to Shaw taurodontism is classified into hypo, meso, and hyper taurodontism on the basis of the relative amount apical displacement of the floor of the pulp chamber, whereas Keene has classified on the basis of 'Taurodont index' where relative height of the pulp chamber to the length of the longest root is taken, while Feichtinger and Rossiwall stated that the distance from the furcation to the amelocementary joint should be greater than the cervico - occlusal distance to define taurodontism. But it is the Shifman and Chanannel who gave a more objective method on the basis if the distance (a) from the lowest point (A) at the occlusal end of the pulp chamber to the highest point at the apical end of the chamber (B), divided by the distance (b) from the occlusal end of the pulp chamber to the apex $(\mathrm{C})$ is $0.2 \mathrm{~mm}$ or greater i.e. $\{\mathrm{A} / \mathrm{b}=0.2 \mathrm{~mm}\}$ and if the distance from the highest point of the pulp chamber floor to cemento-enamel junction is more than $2.5 \mathrm{~mm}^{4,5}$.

This paper presents a case report of generalised taurodontism affecting both deciduous and permanent dentition. 


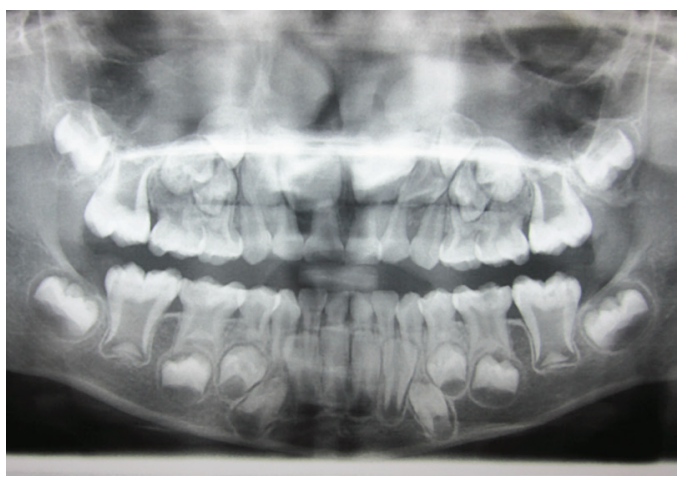

Figure 1 OPG of the patient showing multiple bilateral taurodontic teeth in maxillary and mandibular arch.

\section{Case Report}

A seven year old male patient reported to the Department of Pedodontics and Preventive Dentistry, College of Dental Sciences, Davangere, Karnataka, India with a chief complaint of pain in lower left back region of the jaw since 15 days. The pain was sharp, intermittent, localised and increased on lying down with no aggravating or relieving factors. Medical history and family history was not significant and no abnormality was detected on extra oral examination.

On intraoral examination the child was in mixed dentition phase with deep occlusal caries with 75 \& 85 . Radiographic examination of the same revealed deep carious lesion in relation to 75 and 85 . Interestingly, there were large pulp chambers and small roots present in relation to teeth $74,75,36,84,85 \& 46$. Hence a digital OPG was advised which showed even the primary maxillary molars \& first permanent molars of both the quadrant to be involved (figure 1). Multivisit pulpectomy procedure was done in relation to 75 . The access for endodontic treatment was easily gained but the problem arises with locating the orifices of canals as these teeth have large pulp chambers with rudimentary roots. The presence of taurodontism was confirmed during the clinical procedure as the pulp chamber was large and filled entirely with pulp tissue. The pulp chamber and canals were thoroughly debrided, shaped and obturated with vitapex (Figure 2) followed by placement of stainless steel crown (Figure 3 ) whereas indirect pulp capping was carried out in relation to 85 with light cured .

\section{Discussion}

Taurodontism is an enlargement of the pulp chamber of a multirooted tooth characterized by large pulp chambers and short roots. It was first reported in the remnants of

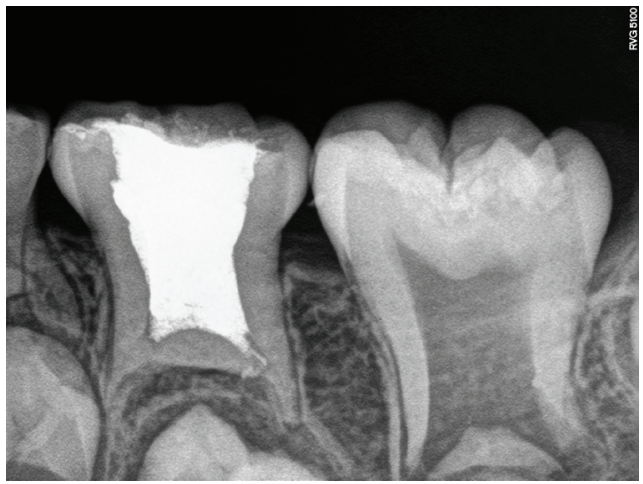

Figure 2 Intraoral periapical radiograph shows endodontically treated 75 with large pulp chamber suggestive of taurodontism

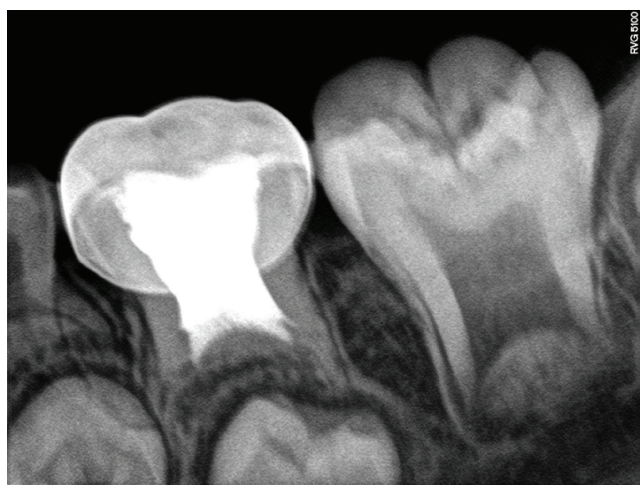

Figure 3 Intraoral periapical radiograph shows stainless steel crown with 75 .

prehistoric hominids ${ }^{4}$. Taurodontism is seen as an isolated trait or as a feature associated with multiple syndromes.

It may occur unilaterally/ bilaterally in both the dentition of same patient, there may be occurrence in family. Permanent dentition is more commonly affected than primary dentition. It has no gender predilection ${ }^{6}$. The teeth most frequently affected are the molars, whereas premolars present a lower incidence and the mandibular teeth more commonly affected than the upper teeth ${ }^{7}$. In the present case the patient had normal development and could not be associated to any syndrome. As seen in OPG we can appreciate taurodontism with all primary molars \& first permanent molars (Figure 1). Diagnosis of the same was done on basis of routine radiographs. Thus the present case is a Non- syndormic taurodontism involving both the dentition.

Taurodontism may be genetically transmitted and could be associated with an increased number of $\mathrm{X}$ chromosomes. 
In certain situations, identification of taurodontism may be helpful in early recognition of Klinefelter syndrome thus helpful in substantially improving the quality of life. As Taurodontism clinically appears as a normal tooth, the diagnosis of taurodontism is usually a subjective determination made from diagnostic radiographs. Therefore the differential diagnoses of taurodontism are pseudohypoparathyroidism, hypophosphatasia, dentinogenesis-imperfecta, hypophosphataemia and vitamin D-resistant dependent rickets. Sometimes young permanent tooth may also be mistaken for taurodontism but can be easily differentiated as it has wide apical foramina ${ }^{3}$.

An endodontic procedure in taurodontic teeth is a complex and difficult procedure as it is associated with increased incidence of haemorrhage during access opening which many a times is mistaken for perforation. However like said earlier the access for endodontic treatment can easily be gained but difficulty arises in locating the orifices of the canals. Hence, careful exploration of the grooves between all orifices with the help of magnification is highly recommended to reveal additional orifices and canals. As the pulp of a taurodontic tooth is usually voluminous, to ensure complete debridement of the pulp, $2.5 \%$ sodium hypochlorite has been suggested initially as an irrigant to dissolve pulp tissue ${ }^{4}$. The preferred material for obturation is vitapex not the commonly used zinc oxide eugenol as its rate of resorption is slower than the root which may lead to delayed exfoliation of deciduous teeth. The endodontic treatment in primary teeth is further complicated due to various levels of resorption of their small roots ${ }^{3}$. In the present case, the treatment carried out for 85 is indirect pulp capping with light cure GIC whereas multivisit pulpectomy with vitapex was carried out in relation to 75 followed by a stainless steel crown.

\section{Conclusion}

Every clinician must exercise vigilance when treating a taurodontic tooth. These teeth are associated with numerous complications withwide variations in size and shape of the pulp chamber with varying degrees of obliteration and canal configuration. Hence, early identification of taurodonts through radiographs and rendition of preventive care is of utmost importance.

\section{References}

1. Manjunatha, B. S., and Suresh Kumar Kovvuru. "Taurodontism-A Review on its etiology, prevalence and clinical considerations." J Clin Exp Dent 2010 :( 2.4):187-90.

2. Nagaveni NB, Radhika NB. Prevalence of taurodontism in primary mandibular first molars of ethnic Indian Children. Gen Dent 2012;60(5):e3 $35-40$.

3. Bafna Y, Kambalimath HV, Khandelwal V, Nayak P. Taurodontism in deciduous molars. BMJ Case Rep. 2013 Jun 3;2013.

4. Jafarzadeh H, Azarpazhooh A, Mayhall JT. Taurodontism: a review of the condition and endodontic treatment challenges. Int Endod J. 2008 May;41(5):375-88.

5. Panigrahi A, Panigrahi RG, K T S, Bhuyan R, Bhuyan SK. Non syndromic familial bilateral decidious taurodontism - a first case report. J Clin Diagn Res. 2014 Dec;8(12).

6. Gedik R, Cimen M. Multiple taurodontism: report of case. ASDC J Dent Child. 2000 May-Jun;67(3):216-7.

7. Tiku A, Damle SG, Nadkarni UM, Kalaskar RR. Hypertaurodontism in molars and premolars: management of two rare cases. J Indian Soc Pedod Prev Dent. 2003 Dec;21(4):131-4.

How to cite this article:

Bharath .KP, Priya, Poornima .P, Neena I.E. Non Syndromic Generalised Taurodontism in Primary and Permanent teeth -A Case Report. CODS J Dent 2015;7: 90-92

Source of support: Nil. Conflict of interest: None Declared. 